\title{
LINEAR FUNCTIONALS AND INTEGRALS IN ABSTRACT SPACES ${ }^{1}$
}

\author{
H. H. GOLDSTINE
}

In his addendum to Saks, Theory of the Integral, Banach ${ }^{2}$ considers a Lebesgue integral defined in a manner quite similar to that of Daniell $^{3}$ and remarks that no use is made of a measure. It is, however, quite easy to show that Banach's and Daniell's integrals are expressible as Lebesgue integrals whose measure functions are regular outer measures in the sense of Carathéodory.

In the first two sections a linear, non-negative functional is considered. Upper and lower functionals are associated with this functional, and by means of them inner and outer measures are defined. It is shown that if the inner and outer measures of a set coincide, the set is measurable. To establish the converse a continuity assumption is made in $\$ 3$, and a representation theorem in terms of the Lebesgue integral is obtained. It is shown in $\$ 4$ that the theorem of Lebesgue for term-wise integration holds for semi-uniformly convergent $\mathbb{R}$ systems.

1. Preliminary definitions. It will be convenient to consider two linear classes of real-valued functions defined on a completely arbitrary range $\mathfrak{P}$. The first of these sets will be symbolized by $\mathfrak{X}$ and it will be supposed to contain the absolute value of every function in it. The second set $\$$ is made up of all functions $z(p)$ such that for some $x$ in $\mathfrak{X},|z| \leqq x$. In accordance with Daniell's notation the symbols $x_{1} \bigvee x_{2}, x_{1} \wedge x_{2}$ represent the larger and smaller, respectively, of the functions $x_{1}, x_{2}$ at each place $p$. Both these functions are in $\mathfrak{X}$ since $x_{1} \bigvee x_{2}=x_{2}+\left(x_{1}-x_{2}\right) \bigvee 0, x_{1} \wedge x_{2}=x_{1}-\left(x_{1}-x_{2}\right) \bigvee 0$ and since $x \vee 0$ $=(x+|x|) / 2$.

Throughout this paper we shall be concerned with a linear functional $I$ which is defined on the class $\mathfrak{X}$ and is non-negative in the sense that $x \geqq 0$ implies $I(x) \geqq 0$. Associated with this functional $I$ there are two others $I^{*}, I_{*}$ which will be called the upper and lower functionals and are defined by the equations

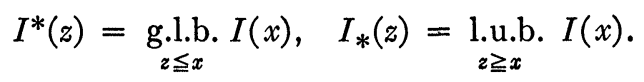

\footnotetext{
1 Presented to the Society, April 13, 1940.

2 Op. cit., p. $320 \mathrm{ff}$.

${ }^{3}$ A general form of integral, Annals of Mathematics, vol. 19 (1918), pp. 279-294.
} 
For functionals defined on other linear subspaces of 3 similar upper and lower functionals may be defined, and they will have the same properties as $I^{*}, I_{*}$. It is easy to show that $I_{*}(z)=-I^{*}(-z) ; I_{*}(z) \leqq I^{*}(z)$; $I^{*}\left(z_{1}+z_{2}\right) \leqq I^{*}\left(z_{1}\right)+I^{*}\left(z_{2}\right) ; \quad I_{*}\left(z_{1}+z_{2}\right) \geqq I_{*}\left(z_{1}\right)+I_{*}\left(z_{2}\right) ;$ for $r \geqq 0$ that $I_{*}(r z)=r I_{*}(z), I^{*}(r z)=r I^{*}(z)$; and that $I_{*}, I^{*}$ are non-negative.

LEMMA 1.1. For every $z I^{*}(|z|)-I_{*}(|z|) \leqq I^{*}(z)-I_{*}(z)$.

To prove this result we notice that if $x_{1} \geqq z, x_{2} \geqq-z$, then $x_{1} \bigvee x_{2} \geqq|z|$ and $x_{1} \wedge x_{2} \geqq-|z|$ and $x_{1} \bigvee x_{2}+x_{1} \wedge x_{2}=x_{1}+x_{2}$. We have then $I^{*}(|z|)-I_{*}(|z|) \leqq I\left(x_{1} \bigvee x_{2}\right)+I\left(x_{1} \wedge x_{2}\right)=I\left(x_{1}\right)+I\left(x_{2}\right)$ for all $x_{1}$, $x_{2}$ such that $-x_{2} \leqq z \leqq x_{1}$, from which the conclusion follows at once.

2. An extension of I. In this section we obtain an extension of the range of definition of $I$ and are thereby enabled to introduce inner and outer measures into the discussion. Let $\mathfrak{Y}$ be the set of all $z$ such that $I_{*}(z)=I^{*}(z)$.

THEOREM 1. The set $\mathfrak{Y}$ is a linear subclass of $\mathbb{Z}$ containing $\mathfrak{X}$ and on which there is a linear, non-negative functional $L$ coinciding on $\mathfrak{X}$ with $I$. The set $\mathfrak{Y}$ is such that if $y_{1}, y_{2}$ are in it so are $y_{1} \wedge y_{2}, y_{1} \vee y_{2}$, and it is furthermore such that a function $z$ is in $\mathfrak{Y}$ if and only if the relation

is satisfied.

$$
L_{*}(z) \equiv \underset{z \geqq y}{\text { l.u.b. }} L(y)=L^{*}(z) \equiv \underset{z \leqq y}{\text { g.l.b. }} L(y)
$$

To establish the theorem let $L(y)$ ke the common value of $I^{*}(y)$ and $I_{*}(y)$. It follows quite easily that $L$ is linear and non-negative. To complete the proof let us first show that $L^{*}, L_{*}$ coincide with $I^{*}, I_{*}$. It suffices evidently to show that $L^{*}(z)=I^{*}(z)$. By definition $L^{*}(z) \leqq L(y) \leqq I(x)$ for $z \leqq y \leqq x$ and hence $L^{*}(z) \leqq I^{*}(z)$. It also follows from the definition of $L^{*}$ that there is a sequence $y_{n} \geqq z$ such that $L^{*}(z)>L\left(y_{n}\right)-1 / n=I^{*}\left(y_{n}\right)-1 / n \geqq I^{*}(z)-1 / n$ for every integer $n$, which shows that $L^{*}(z) \geqq I^{*}(z)$. It remains now only to show that $|y|$ is in $\mathfrak{Y}$, and this follows readily from Lemma 1.1 .

For convenience let us call a set $E$ an $M$-set or say it is in $\mathfrak{M}$ in case its characteristic function is in $\mathfrak{V}$, and let us assume hereafter that the function $z(p)=1$ is in $\mathfrak{Y}$. We may then define the measure $m E$ of an arbitrary set $E$ as the lower bound of $L\left(y_{F}\right)$ for all $M$-sets $F$ containing $E, y_{F}$ being the characteristic function of $F$, and we define the lower measure $m_{*} E$ in an analogous way. ${ }^{4}$

${ }^{4}$ In his thesis, E. T. Welmers has considered similar definitions of measures. See Set Functions and Measurability Conditions, University of Michigan doctoral dissertation, 1936. 
THEOREM 2. The functions $m_{*}, m$ are monotone increasing set-functions such that $m_{*} E \leqq m E$ and for every finite number of sets $E_{n}$

$$
m\left(\sum E_{n}\right) \leqq \sum m E_{n}, \quad m_{*}\left(\sum E_{n}\right) \geqq \sum m_{*} E_{n} .
$$

$A$ set $E$ is an $M$-set if and only if $m_{*} E=m E$.

The first parts of the theorem are easily proved, and it is clear that if $E$ is an $M$-set then $m_{*} E=m E$. Conversely if $m_{*} E=m E$, there is a sequence of $M$-sets $F_{n} \subset E$ such that $L^{*}\left(z_{E}\right)<L\left(y_{F_{n}}\right)+1 / n \leqq L_{*}\left(z_{E}\right)$ $+1 / n$, since $L^{*}\left(z_{E}\right) \leqq m E$. Hence $L_{*}\left(z_{E}\right)=L^{*}\left(z_{E}\right)$.

TheOREM 3. Every $M$-set is measurable in the usual sense.

To prove this result we note first that the set $\mathfrak{M}$ is restrictedly additive. It is then clear that $m X \cdot E+m X \cdot C E \leqq L\left(y_{F \cdot E}+y_{F \cdot C E}\right)=L\left(y_{F}\right)$ for every set $X$ and every $M$-set $F \supset X$ and hence $m X \cdot E+m X \cdot C E$ $\leqq m X$, from which it follows that $E$ is measurable.

3. A continuity assumption. To proceed further it is desirable to make a continuity assumption. We assume that for every monotone decreasing sequence of non-negative functions $x_{n}$ in $\mathfrak{X}, I_{*}\left(\lim x_{n}\right)$ $\geqq \lim I\left(x_{n}\right)$. An obvious but important corollary of this hypothesis is that the function $\left(\lim x_{n}\right)$ is in the set $\mathfrak{Y}$, provided that the sequence $x_{n}$ is as described in the preceding sentence.

LEMмA 3.1. The limit of a monotone decreasing sequence of nonnegative functions $y_{n}$ in $\mathfrak{Y}$ is again in $\mathfrak{Y}$, and $L\left(\lim y_{n}\right)=\lim L\left(y_{n}\right)$.

To prove this lemma we recall that there is a double sequence of non-negative functions $x_{m n}$ in $\mathfrak{X}$ such that $x_{m n} \leqq y_{n}$ and $I_{*}\left(y_{n}\right)<I\left(x_{m n}\right)$ $+1 / m 2^{n}$. There is no loss in generality if we assume that for each $n$ the sequence $x_{m n}$ is monotone increasing in $m$ since $\bar{x}_{1 n}=x_{1 n}, \bar{x}_{m n}=\bar{x}_{m-1, n}$ $\bigvee x_{m n}$ has the desired monotone property and $I_{*}\left(y_{n}\right)<I\left(\bar{x}_{m n}\right)+1 / m 2^{n}$ since $x_{m n} \leqq \bar{x}_{m n}$. We show next that there is another sequence of nonnegative functions $\xi_{m n}$ in $\mathfrak{X}$ which is monotone decreasing in $n$, monotone increasing in $m$ and for which $\xi_{m n} \leqq x_{m n}$. This sequence may be defined as follows: $\xi_{m 1}=x_{m 1}, \xi_{m n}=\xi_{m, n-1} \wedge x_{m n}$. By a proof like that given by Daniell ${ }^{5}$ it then follows that $L\left(y_{n}\right)<I\left(\xi_{m n}\right)+1 / m$. With the help of the inequalities $\lim _{n} \xi_{m n} \leqq \lim _{n} y_{n} \leqq y_{n}$, it is seen that $L\left(\lim _{n} \xi_{m n}\right) \leqq L_{*}\left(\lim y_{n}\right) \leqq L^{*}\left(\lim y_{n}\right) \leqq \lim L\left(y_{n}\right) \leqq \lim _{n} I\left(\xi_{m n}\right)+1 / m$ $\leqq L\left(\lim _{n} \xi_{m n}\right)+1 / m$; whence $L_{*}\left(\lim y_{n}\right)=L^{*}\left(\lim y_{n}\right)$ since $L\left(\lim _{n} \xi_{m n}\right)$ is monotone increasing and bounded.

5 Daniell, loc. cit., p. $283 \mathrm{ff}$. 
LEMMA 3.2. To every set $E$ there corresponds an $M$-set $F \supset E$ such that $m E=m F$ and $m(F-E)=m E-m_{*} E$.

According to the definition of $m$ there is a sequence $F_{n}$ of $M$-sets each containing $E$, such that $m E>L\left(y_{F_{n}}\right)-1 / n$. With the help of the continuity hypothesis made above it then follows easily that $F=\prod F_{n}$ is in $\mathfrak{M}$ and that $L\left(y_{F}\right) \geqq m E \geqq L\left(y_{F}\right)=m F$. To prove the second part of the lemma one needs only to keep in mind Theorem 3 and make a proof quite like that of Carathéodory. ${ }^{6}$

THEOREM 4. The class $\mathfrak{M}$ is completely additive and contains every measurable set $E$. The set-function $m$ is a regular outer measure of Carathéodory.

It is quite easy to show that the intersection of a denumerable number of sets in $\mathfrak{M}$ is again in $\mathfrak{M}$, and this fact coupled with the restricted additivity of $\mathfrak{M}$ suffices to prove that $\mathfrak{M}$ is completely additive. By the lemma just proved there is a set $F$ in $\mathfrak{M}$ that contains $E$ and for which $m F=m E$. Since $E$ is measurable, we have $m F=m E$ $+m(F-E)$ and hence $m(F-E)=0$, which implies that $m_{*} E=m E$.

Before proceeding further with our proof let us show that if $F_{n}$ is a monotone increasing sequence of measurable sets, then $\lim m F_{n}$ $=m\left(\lim F_{n}\right)$. To prove this statement let $F=\lim F_{n}$ and notice that the characteristic functions of the sets $F-F_{n}$ form a monotone decreasing sequence converging to zero. Hence $L\left(y_{F}\right)-L\left(F_{n}\right)=m F-m F_{n}$ converges to zero.

The regularity of $m$ follows from Lemma 3.2 and Theorem 3 . If $\left\{E_{n}\right\}$ is an arbitrary sequence of sets, there is another sequence of measurable hulls $F_{n} \supset E_{n}$. It is thus true that $m\left(\sum E_{i}\right) \leqq m\left(\sum F_{i}\right)$ $\leqq \sum m F_{i}=\sum m E_{i}$ and hence $m$ is an outer measure.

ThEOREM 5. Every $y$ in $\mathfrak{Y}$ is integrable and

$$
L(y)=\int y d m \text {. }
$$

Conversely every integrable function is in $\mathfrak{V}$.

It follows at once from the definitions of $m$ and the integral that (3.1) holds when $y$ is a simple function, and from Lemma 3.1 that $\lim L\left(y_{n}\right)=L\left(\lim y_{n}\right)$ for monotone increasing sequences for which $\left(\lim y_{n}\right)$ is in $\mathfrak{Y}$. It evidently suffices to restrict out attention to nonnegative functions $y$, recall that they are limits of monotone increas-

6 Vorlesungen über reelle Funktionen, p. 262, Theorem 5. 
ing sequences of simple functions, and use Lebesgue's theorem on the integration of monotone sequences.

To prove the converse let $z$ be an arbitrary integrable function. There is then a monotone decreasing sequence $\left\{y_{n}\right\}$ of functions in $\mathfrak{V}$ converging to $z$ for which the relation

$$
\lim _{n} L\left(y_{n}\right)=L^{*}(z)
$$

holds. With the help of Lebesgue's theorem on term-wise integration, the equation (3.1) and the relation (3.2) we have at once the equality of $L^{*}(z)$ and the integral of $z$. In a quite similar fashion it follows that $L_{*}(z)$ is equal to the integral of $z$ and thus to $L^{*}(z)$, from which it follows that $z$ is in $\mathfrak{Y}$.

To obtain the following corollary it is convenient to let $\mathfrak{U}$ be a linear space of real-valued functions $u$ on $\mathfrak{P}$ containing $|u|$ and $u(\mathfrak{B})=1$.

COROLlARY 1. Let $I$ be a linear and non-negative functional defined on $\mathfrak{U}$ and let it be such that $I\left(u_{n}\right)$ converges to zero whenever $\left\{u_{n}\right\}$ is a monotone decreasing sequence with limit zero. There is then a regular outer measure of Caratheodory such that

$$
I(u)=\int u d m, \quad u \in \mathfrak{U} .
$$

By a method quite like that of Daniell ${ }^{7}$ we may extend $I$ to be defined over a space $\mathfrak{X}$ which has the properties described in the first paragraphs of $\$ 1$ and $\S 3$. Our corollary then follows immediately from the theorem.

4. The theorem of Lebesgue. Let $\&$ be an arbitrary class of elements $l$ and let $R$ be a transitive binary relation between the elements of $\mathfrak{R}$, having the so-called compositive property which guarantees the existence of an element in the $R$-relation to each of a pair of preassigned elements. An $R R$-system of numbers is then defined as a realvalued function $a_{l}$ defined on $\&$.

Recalling Theorem 5 , we see that $\mathfrak{V}$ is exactly the space of all integrable functions. If $y_{l}$ is an $\Omega R$-system of integrable functions converging almost everywhere to an integrable $y$, then without imposing more stringent convergence properties, we could not hope to prove that the equation

$$
\lim _{l} \int y_{l} d m=\int y d m
$$

\footnotetext{
${ }^{7}$ Daniell, loc. cit., pp. 285-287.
} 
is valid. Suppose, for the moment, that every finite set of points has measure zero and that the measure of $\mathfrak{B}$ is different from zero. If we choose $l$ to be a finite subset of $\mathfrak{P}$ and $y_{l}(p)$ to be one for $p$ in $l$ and zero elsewhere, then $y_{l}$ evidently converges to one, and yet the equation (4.1) does not hold.

The system $y_{l}$ is said to converge semi-uniformly ${ }^{8}$ to $y$ in case to each $e>0$ and $p$ there is an $l_{e p}$ such that the inequality

$$
|y \iota(p)-y(p)|<e
$$

is satisfied by all $l R l_{e p}$ and in case for each fixed $e$ the set of elements $l_{e p}$, as $p$ varies, is at most denumerable.

THEOREM 6. Let $y_{l}$ be an $\Omega R$-system of measurable functions such that for all $l$ in the $R$-relation to some $l_{0}$ the inequality $\left|y_{l}(p)\right| \leqq y_{0}(p)$ holds, where $y_{0}$ is integrable, Then if $y_{l}$ converges almost everywhere in a semi-uniform way to a measurable function $y$, the relation (4.1) is valid.

It follows from the definition of semi-uniform convergence that there is a monotone increasing sequence $l_{n}$ and a system of integers $n_{e p}$ such that the inequality (4.2) is satisfied for $e>0$ and $l R l_{n}$ when $n=n_{e p}$. It is then evident that if $l_{1 n} R l_{n}(n=1,2, \ldots)$ the sequence obtained from $y_{l}$ by setting $l=l_{1 n}$ will converge to $y$, and then by Lebesgue's theorem for sequences we have

$$
\lim _{n} \int y_{l_{1 n}} d m=\int y d m .
$$

If our theorem were false, there would be a sequence $l_{1 n} R l_{n}$ such that

$$
\left|\int\left(y_{l_{1 n}}-y\right) d m\right| \geqq e,
$$

which contradicts (4.3).

UNIVERSITY OF MICHIGAN

8 E. H. Moore, General Analysis, Part II, p. 41 and p. 49. 